\title{
Role of aldo-keto reductases and other doxorubicin pharmacokinetic genes in doxorubicin resistance, DNA binding, and subcellular localization
}

Allan D Heibein ${ }^{1}$, Baoqing Guo ${ }^{2}$, Jason A Sprowl $\left.\right|^{3}$, David A MacLean ${ }^{1,4}$ and Amadeo M Parissenti $1,2,3,4,5^{*}$

\begin{abstract}
Background: Since proteins involved in chemotherapy drug pharmacokinetics and pharmacodynamics have a strong impact on the uptake, metabolism, and efflux of such drugs, they likely play critical roles in resistance to chemotherapy drugs in cancer patients.

Methods: To investigate this hypothesis, we conducted a whole genome microarray study to identify difference in the expression of genes between isogenic doxorubicin-sensitive and doxorubicin-resistant MCF-7 breast tumour cells. We then assessed the degree of over-representation of doxorubicin pharmacokinetic and pharmacodynamic genes in the dataset of doxorubicin resistance genes.

Results: Of 27,958 Entrez genes on the array, 7.4 per cent or 2,063 genes were differentially expressed by $\geq 2$-fold between wildtype and doxorubicin-resistant cells. The false discovery rate was set at 0.01 and the minimum $p$ value for significance for any gene within the "hit list" was 0.01 . Seventeen and 43 per cent of doxorubicin pharmacokinetic genes were over-represented in the hit list, depending upon whether the gene name was identical or within the same gene family, respectively. The most over-represented genes were within the $1 \mathrm{C}$ and $1 \mathrm{~B}$ families of aldo-keto reductases (AKRs), which convert doxorubicin to doxorubicinol. Other genes convert doxorubicin to other metabolites or affect the influx, efflux, or cytotoxicity of the drug. In further support of the role of AKRs in doxorubicin resistance, we observed that, in comparison to doxorubicin, doxorubincol exhibited dramatically reduced cytotoxicity, reduced DNA-binding activity, and strong localization to extra nuclear lysosomes. Pharmacologic inhibition of the above AKRs in doxorubicin-resistant cells increased cellular doxorubicin levels, restored doxorubicin cytotoxicity and re-established doxorubicin localization to the nucleus. The properties of doxorubicinol were unaffected.

Conclusions: These findings demonstrate the utility of using curated pharmacokinetic and pharmacodynamic knowledge bases to identify highly relevant genes associated with doxorubicin resistance. The induction of one or more of these genes was found to be correlated with changes in the drug's properties, while inhibiting one specific class of these genes (the AKRs) increased cellular doxorubicin content and restored drug DNA binding, cytotoxicity, and subcellular localization.
\end{abstract}

Keywords: Doxorubicin, Resistance, Gene profiling, PharmGKB, Pharmacokinetics, Aldo-keto reductases, Cytotoxicity, Lysosome, DNA-binding, Drug localization

\footnotetext{
* Correspondence: aparissenti@hsnsudbury.ca

${ }^{1}$ Graduate Program in Biology, Laurentian University, Sudbury, ON, Canada

${ }^{2}$ Regional Cancer Program, Sudbury Regional Hospital, Sudbury, ON P3E 5J1,

Canada

Full list of author information is available at the end of the article
}

\section{Ciomed Central}

(c) 2012 Heibein et al.; licensee BioMed Central Ltd. This is an Open Access article distributed under the terms of the Creative Commons Attribution License (http://creativecommons.org/licenses/by/2.0), which permits unrestricted use, distribution, and reproduction in any medium, provided the original work is properly cited. 


\section{Background}

Doxorubicin is a DNA-binding, topoisomerase II inhibitor [1,2], which is among the most effective chemotherapy drugs in cancer treatment $[1,3]$. However, intrinsic or acquired resistance to doxorubicin in patient tumours is common, resulting in treatment failure and disease progression. Multiple mechanisms for doxorubicin resistance have been identified in vitro, such as the increased expression of drug transporters [4-7], alterations in doxorubicin metabolism [8] or localization $[9,10]$, and defects in the drug's ability to induce apoptosis [11]. Unfortunately, progress in restoring drug sensitivity for drug-resistant tumours, particularly by inhibiting drug efflux transporters, has been incremental at best $[12,13]$. This limited progress demands that a more nuanced approach be taken, including the identification of all proteins that likely affect the pharmacokinetics and pharmacodynamics of doxorubicin.

Genome profiling is a method that can provide data on gene expression and/or allelic variations across biological samples, often using whole genome approaches. This promises to be a great aid to oncologists in identifying and treating drug-resistant tumours. Unfortunately, this task is a difficult one, given the variability associated with patient data sets and the large number of "false positives" inherent in such approaches from by-stander effects. One method to improve the identification of genes relevant to a specific phenomenon such as doxorubicin resistance is to pair knowledge of metabolic or signal transduction pathways to gene expression data [14]. In this study, we use full genome microarray analysis to compare gene expression between MCF-7 cells selected for maximal resistance to doxorubicin (MCF$7_{\text {DOX2-12 }}$ cells) and equivalent cells selected for the same number of passages in the absence of drug $\left(\mathrm{MCF}-7_{\mathrm{CC} 12}\right.$ cells). After identifying genes having altered expression in doxorubicin-resistant cells, we then used a well-known, curated pharmacogenomics knowledgebase (PharmGKB) to identify which of these genes play a role in doxorubicin pharmacokinetics or pharmacodynamics, as these were more likely to have a direct effect on doxorubicin efficacy. This combination of full genome microarray analysis identifying genes differentially expressed upon acquisition of doxorubicin resistance with an assessment of overrepresentation of doxorubicin pharmacokinetic or pharmacokinetic genes in the dataset provided significant insight into new pathways associated with doxorubicin resistance. Moreover, extensive comparisons between the biochemical properties of doxorubicin and one of its metabolites (doxorubicinol) provided us with significant insight into how a simple hydroxylation reaction can strongly affect the biochemical and cellular properties of doxorubicin, including dramatically reduced cytotoxicity, diminished DNA- binding activity, altered cellular accumulation of the drug and altered subcellular localization.

\section{Results \\ Differentially expressed genes upon acquisition of doxorubicin resistance}

Using full genome Agilent microarrays and Partek Genomics Suite, 2063 genes from a total of 27958 Entrez genes on the array (7.4\%) were found to be differentially expressed by $\geq 2$-fold between $\mathrm{MCF}-7_{\mathrm{CC} 12}$ cells MCF-7 ${ }_{\text {DOX2-12 }}$ cells. The false discovery rate was set at 0.01 and the minimum $\mathrm{p}$ value for significance for any gene within the "hit list" was 0.01 . The microarray data was deposited in the NCBI Gene Expression Omnibus (GEO) database, accession number GSE27254) in accordance with MIAME standards [15]. Access to the microarray data can be obtained via the following url: http://www.ncbi.nlm.nih.gov/geo/query/acc.cgi?token= dbezngycywquuhm\&acc $=$ GSE27254.

The identification of thousands of genes changing expression upon selection of MCF-7 cells for doxorubicin resistance was similar to the numbers of genes observed when these cells were selected for resistance to other chemotherapy agents (data not shown). These findings indicate that a significant amount of the transcriptome appears altered as these cells are selected for doxorubicin resistance. In addition to providing candidate genes that may be involved in doxorubicin resistance, the microarray data served to demonstrate that MCF-7 ${ }_{\text {DOX2 }}$ cells at selection dose 12 and MF-7 $7_{\mathrm{CC}}$ cells (selected to the same passage number in the absence of doxorubicin) are isogenic, since the vast majority of genes (92.6\%) differed in expression by $<2$-fold between the two cell lines [see statistical analysis of microarray (SAM) plots in Figure 1]. This suggests that observed differences in gene expression are likely related to the acquisition of doxorubicin resistance and not simply a selection for a rare, unrelated cell type within the cell population.

In examining the identities of genes exhibiting the greatest changes in expression upon acquisition of doxorubicin resistance, a number of these genes play a role in doxorubicin metabolism. Consequently, we assessed the extent of "over-representation" of doxorubicin metabolism genes by comparing the names of differentially expressed genes in the microarray "hit list" with those listed in a curated list of genes associated with doxorubicin pharmacokinetics or pharmacodynamics in tumour cells and cardiomyocytes available on the Pharmacogenetics Knowledge Base (PharmGKB) [16]. This list can be found at the url: http://www.pharmgkb.org/drug/ PA449412\#tabview $=$ tab5\&subtab $=33$ and is depicted in Additional file 1: Table S1. Figure 2 shows two pathway 


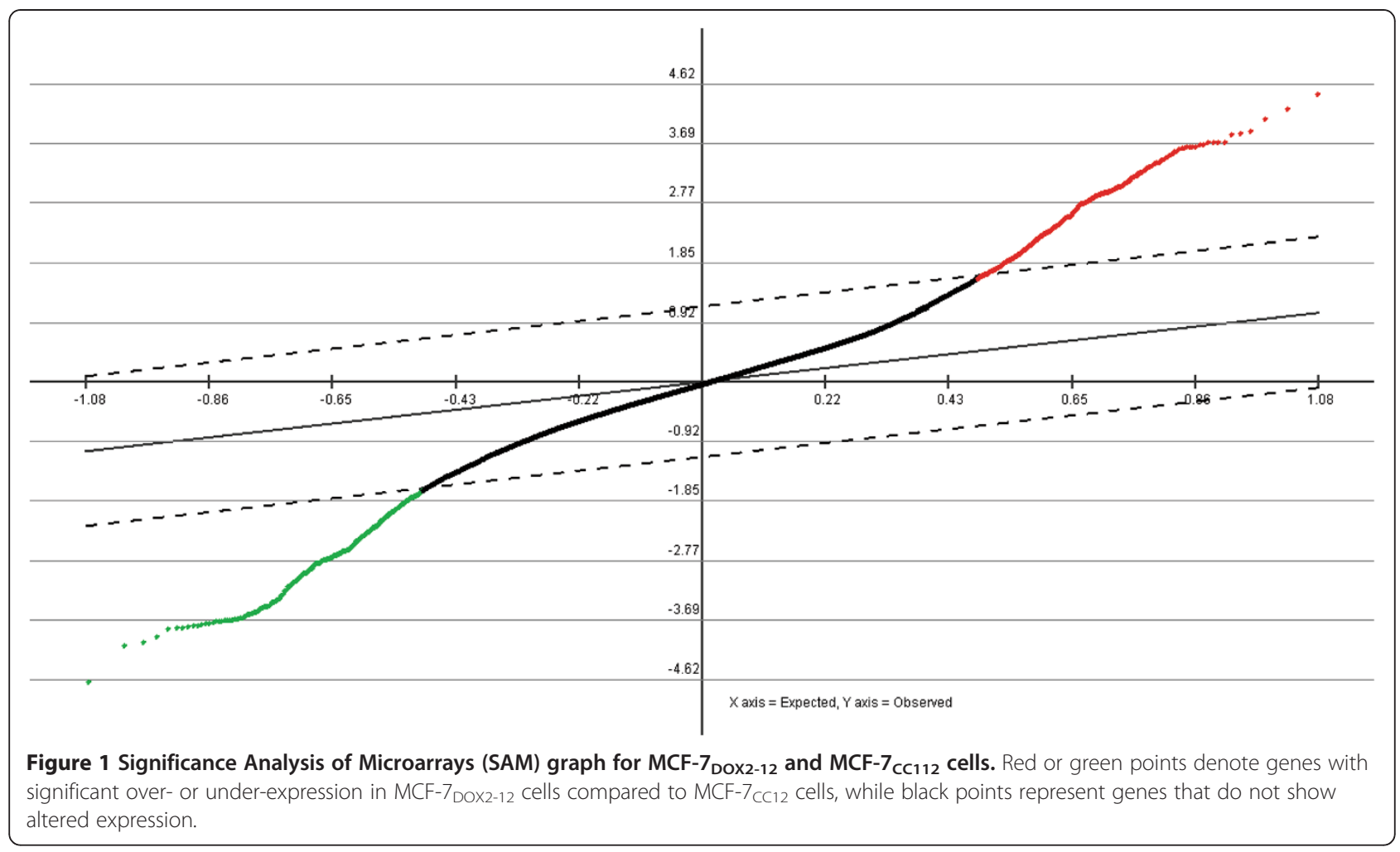

diagrams available through the PharmGKB website that document the different proteins that impact on the uptake, metabolism, and efflux of doxorubicin in cardiomyocytes (A) and tumour cells (B). A comparison of a list of these proteins (Additional file 1: Table 1) with the list of genes significantly changed by $\geq 2$-fold in doxorubicin-resistant cells in the above microarray experiment $(p \leq 0.01)$ revealed that doxorubicin pharmacokinetic and pharmacodynamic genes are highly over-represented in the list of differentially expressed genes. Identical genes or genes having the same family name on both lists are depicted in bold, with the fold increase $(+)$ or decrease (-) in expression in the microarray experiment listed beside each gene. Additional file 2: Table S2 depicts the results of our over-representation analysis. At a false discovery rate of $0.01,8$ of the 46 genes listed in the doxorubicin pharmacokinetics/ pharmacodynamics pathways $(17 \%)$ were direct matches and 20 or $43 \%$ were partial matches (same gene family). The $\mathrm{p}$ value for significance of this over-representation relative to randomly selected genes was 0.05 for identical matches and $<0.0001$ for either identical or partial matches. Since these genes directly affect the uptake, efflux, metabolism or cytotoxicity of doxorubicin, they have a strong potential to play a role in doxorubicin resistance. The identities of these genes provide a compelling view of the various mechanisms that likely play a role in the acquisition of doxorubicin resistance in breast tumour cells in vitro (see discussion).

\section{Several AKRs are over-expressed in MCF-7 Dox2-12 cells} As previously demonstrated using a much smaller microarray platform (1720 genes) [17], the "1C" family of AKRs was observed to be over-expressed upon acquisition of doxorubicin resistance. Moreover, as shown in Additional file 1: Table S1, a variety of AKR family members were among the most differentially expressed genes upon acquisition of doxorubicin resistance in MCF-7 cells. In these microarray studies, AKR1B1, AKR1B10, AKR1C1, and AKR1C3 all had strongly elevated expression (10.0-, 13.4-, 4.45-, and 4.71-fold, respectively). As stated previously, the product of the AKR family of genes facilitates the conversion of doxorubicin to doxorubicinol [18]. Such a strong overexpression of multiple AKR transcripts in MCF-7 ${ }_{\text {DOX2-12 }}$ cells suggests that the AKRs may play a major role in doxorubicin resistance.

Given that AKR " $1 C$ " isoforms are highly conserved amongst each other [19] and given that, by BLAST analysis, the probes on the Agilent $4 \mathrm{X} 44 \mathrm{~K}$ arrays could not distinguish between the four $1 \mathrm{C}$ transcripts, we designed isoform-specific primers for reverse transcription quantitative polymerase chain reaction (RTqPCR) experiments in order to accurately quantify the levels of expression of 


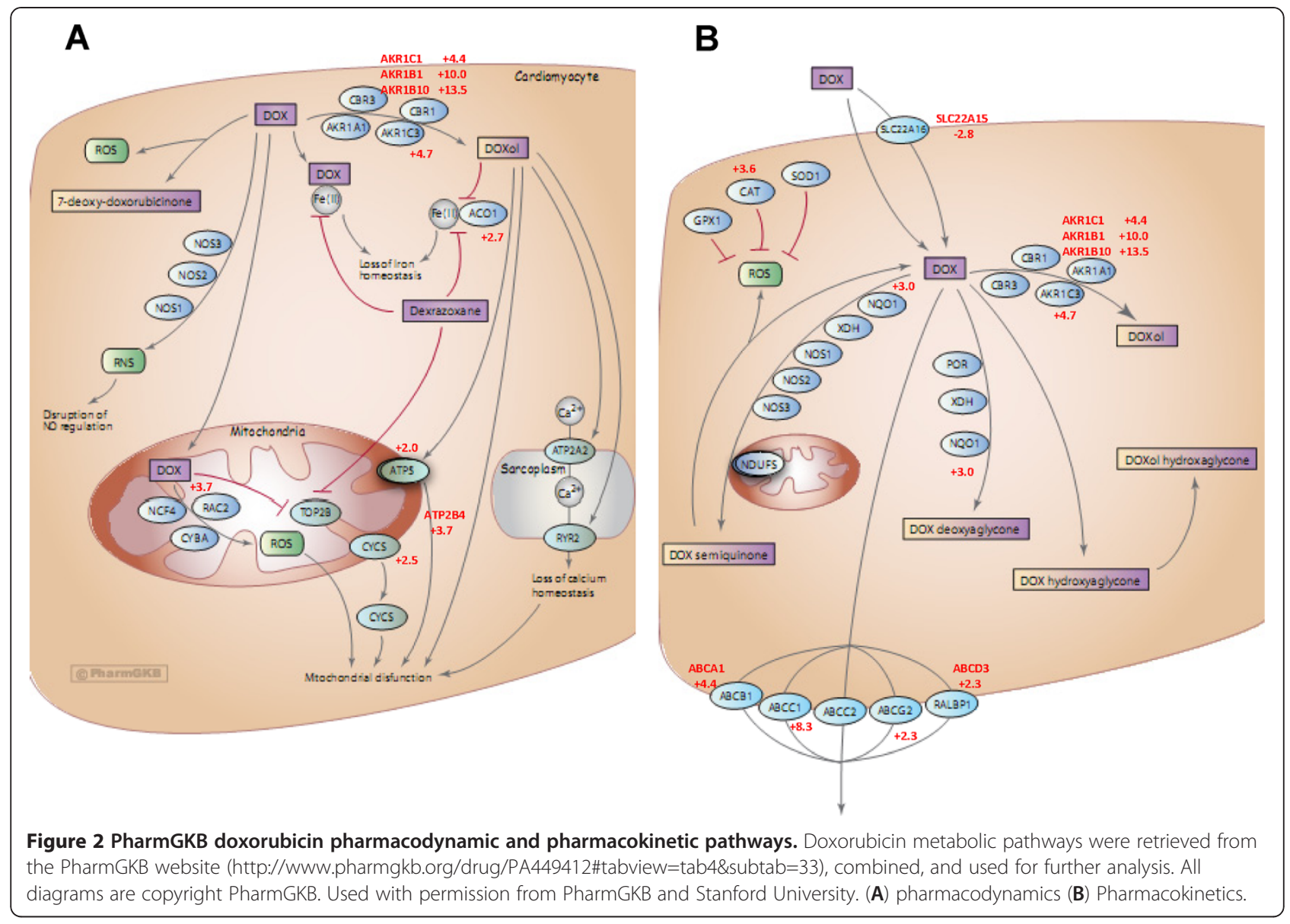

these transcripts. Similarly, since substantial elevations in the highly conserved AKR "1B" isoforms were observed by microarray analysis with AKR1B probes that are not isoform-specific, we also designed isoform-specific RTqPCR primers to accurately quantify transcript levels for the two AKR 1B isoforms identified by microarray analysis. Finally, since the carbonyl reductases (CBRs), like the AKRs, can also play a role in the conversion of doxorubicin to doxorubicinol $[8,20]$, we designed isoform-specific primers to quantify levels of transcripts for two CBR isoforms. The data from these RTqPCR experiments (Figure 3A) revealed that only the $A K R 1 C 2, A K R 1 C 3$ and $A K R 1 B 10$ transcripts were significantly over-expressed in MCF7 DOX2-12 cells compared to MCF-7 $\mathrm{CC12}_{2}$ cells (3.6-, 9.1-, and 10.4-fold, respectively). $C B R 1$ and $C B R 3$ transcripts were not differentially expressed in the doxorubicinresistant cells. As AKR1C3 exhibited one of the highest changes in expression, and since Akr1c3 has been shown to efficiently convert doxorubicin to doxorubicinol [21], we also assessed the expression of Akr1c3 protein in the cell lines. As shown in Figure 3B, immunoblotting experiments confirmed the considerably higher expression of Akr1c3 in MCF-7 1 DOx2-12 cells relative to $\mathrm{MCF}-7_{\mathrm{CC} 12}$ cells.

\section{Doxorubicinol is 1 million-fold less cytotoxic than doxorubicin in MCF-7 cells}

Although it has been previously reported that doxorubicinol is 20 to 27 times less cytotoxic than doxorubicin in fibroblasts or pancreatic tumour cells [22,23], we also wanted to assess in this study the relative sensitivity of MCF-7 ${ }_{\text {DOX2-12 }}$ and MCF-7 ${ }_{\text {CC12 }}$ cells to doxorubicin and doxorubicinol. As shown in Figure 4, the concentration of doxorubicin required to reduce the number of colonies formed in a clonogenic assay by half (the $\mathrm{IC}_{50}$ ) was $7.8 \pm 4.0 \mathrm{nM}$ and $580 \pm 91 \mathrm{nM}$ for $\mathrm{MCF}-7_{\mathrm{CC} 12}$ and MCF7 DOX2-12 cells, respectively, indicating a 74-fold resistance to doxorobucin in MCF-7 ${ }_{\text {DOX2-12 }}$ cells. In contrast, the $\mathrm{IC}_{50}$ of doxorubicinol for the $\mathrm{MCF}-7_{\mathrm{CC} 12}$ cell line was $15 \pm 1.6 \mathrm{mM}$, indicating a reduced cytotoxicity for the metabolite of over 6 orders of magnitude! Interestingly, the cytotoxicity of doxorubicinol was even less in MCF-7 DOX2-12 cells. In fact, we could not achieve sufficient cytotoxcity to compute an $\mathrm{IC}_{50}$ value. Consequently, in our study, doxorubicinol cytotoxicity in a clonogenic assay was dramatically less than doxorubicin, suggesting that the conversion of doxorubicin to doxorubicinol by AKRs or CBRs would essentially eliminate its cytotoxicity in breast tumour cells in culture. 


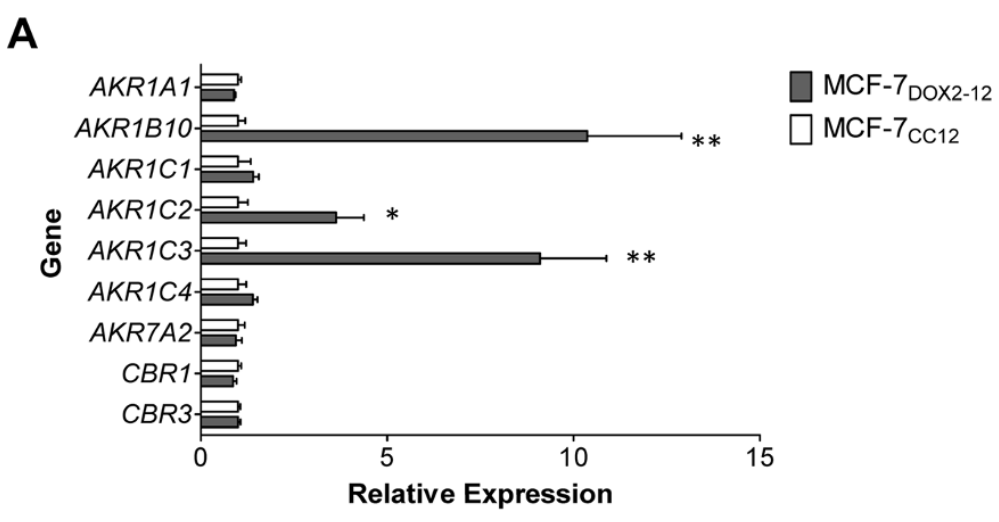

B

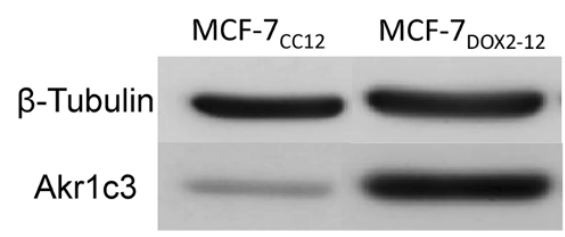

Figure 3 Gene and protein expression levels of doxorubicin to doxorubicinol metabolizing enzymes. (A) Relative changes in expression between MCF-7 ${ }_{C C 12}$ and MCF-7 DOX2-12 cells were assessed by RTaPCR. Each sample was normalized first to RPS28 for loading and then to the average expression of $\mathrm{MCF}-7_{\mathrm{CC} 12}$ cells to determine fold change. ${ }^{*} p \leq 0.05,{ }^{* *} p \leq 0.01$. (B) Western blot analysis of AKR1C3 expression in MCF-7DOX2-12 and MCF-7 CC12 whole cell lysates.

$5 \beta$-cholanic acid restores sensitivity of MCF-7 to doxorubicin

As illustrated in Figure 4, treatment of MCF-7 DOX2-12 cells with both doxorubicin and $5 \beta$-cholanic acid, a potent inhibitor of AKR1B10 [24], AKR1C2, and AKR1C3 activity [25], almost fully restored doxorubicin sensitivity to that of $\mathrm{MCF}-7_{\mathrm{CC} 12}$ cells $\left(\mathrm{IC}_{50}\right.$ of $\left.22.9 \pm 9 \mathrm{nM}\right)$. In contrast, treatment of $\mathrm{MCF}-7_{\mathrm{CC} 12}$ cells with $5 \beta$-cholanic acid and doxorubicin had little effect on doxorubicin sensitivity ( $\mathrm{IC}_{50}$ of $14.1 \pm 8.0 \mathrm{nM}$ ), suggesting insufficient AKR activity in these cells to affect doxorubicin sensitivity. Addition of 5 5 -cholanic acid had no effect on sensitivity of $\mathrm{MCF}-7_{\mathrm{CC} 12}$ cells to doxorubicinol $\left(\mathrm{IC}_{50}\right.$ of $7.5 \pm 10.6 \mathrm{mM})$. However, addition of $5 \beta$-cholanic acid to MCF-7 ${ }_{\text {DOX2-12 }}$ cells did appear to increase their sensitivity to doxorubicinol to a barely detectable range $\left(\mathrm{IC}_{50}\right.$ of $3.2 \pm 1.7 \mathrm{mM}$ ), suggesting a possible ability of the inhibitor to affect further metabolism of doxorubicinol in doxorubicin-resistant cells.

Restoration of doxorubicin sensitivity is accompanied by restored nuclear localization in MCF-7 DOX2-12 $_{\text {Cells }}$

Since doxorubicin is a fluorescent, DNA-binding topoisomerase II inhibitor [1,2], it localizes to the nucleus in

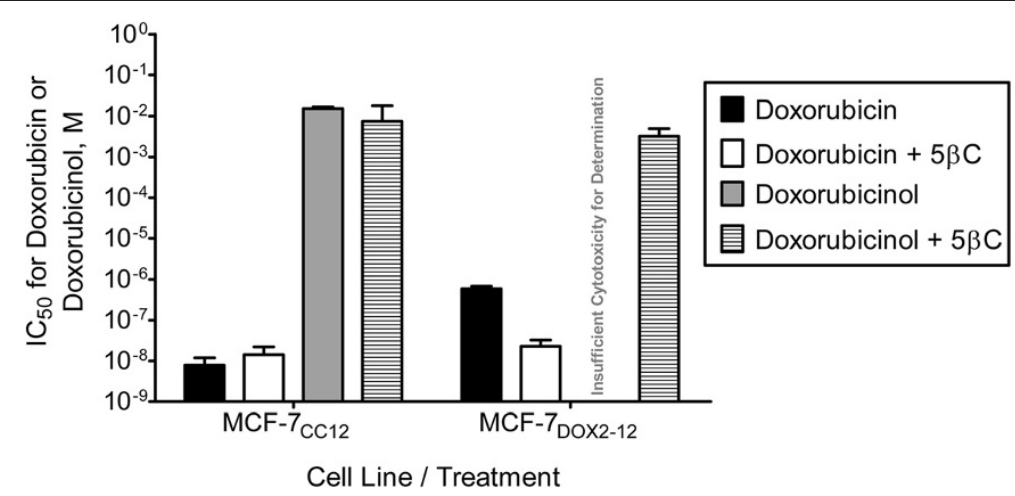

Figure 4 Doxorubicin and doxorubicinol cytotoxicity in MCF-7 $7_{\mathrm{CC} 12}$ and MCF-7 $7_{\mathrm{DOx} 2-12}$ cells upon treatment with or without $5 \beta$-cholanic acid. MCF-7 CC12 $_{2}$ and MCF-7 $7_{\text {DOX2-12 }}$ cells were assessed for their sensitivity to doxorubicin and doxorubicinol in the presence or absence of the AKR1B10, AKR1C2, and AKR1C3 inhibitor 5ß-cholanic acid using a clonogenic assay. 
tumour cells. Drug localization can be effectively monitored by incubating cells with doxorubicin, and removing extracellular drug by extensive washing of the cells, followed by confocal laser scanning fluorescence microscopy [26]. We used this approach to visualize the location of doxorubicin (red fluorescence) in $\mathrm{MCF}-7_{\mathrm{CC} 12}$ and MCF-7 ${ }_{\text {DOX2-12 }}$ cells in the presence of DRAQ5, a highly cell-permeable DNA-binding dye that fluoresces into the infra-red region of the electromagnetic spectrum (shown as pseudo-blue fluorescence). As shown in Figure 5A, we found that fluorescence of $0.5 \mu \mathrm{M}$ doxorubicin localized to nuclei in $\mathrm{MCF}-7_{\mathrm{CC} 12}$ cells, as expected (Figure 5). There was strong co-localization of doxorubicin and DRAQ5 fluorescence (bright purple fluorescence). In MCF-7 DOX2-12 $_{2}$ cells, however, greatly reduced doxorubicin fluorescence was observed, likely due to the reduced uptake of doxorubicin into these cells, as we previously reported [27]. In addition, the little fluorescence that was visible was extra nuclear (as visualized by a lack of co-localization with DRAQ5 staining), and this fluorescence was clustered in the perinuclear region. These observations are consistent with those previously reported by Coley and colleagues for other doxorubicin-resistant cell lines [26]. In previous experiments, we observed that doxorubicin fluorescence in MCF$7_{\text {DOX2-12 }}$ cells co-localized with Lysotracker ${ }^{\text {rux }}$ [17] but not Mitotracker $^{\text {Tix }}$ (Life Technologies, Burlington, VT) staining (data not shown), suggesting that the drug was sequestered in lysosomes and not bound to mitochondrial DNA. The inability of doxorubicin to reach its target (nuclear DNA) can clearly account for the reduced cytotoxicity of doxo-

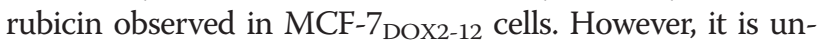
clear whether the perinculear fluorescence exhibited in MCF-7 ${ }_{\text {DOX2-12 }}$ cells was from doxorubicin or perhaps a metabolite of doxorubicin that retains its fluroescence, such as doxorubicinol. As shown in Figure 5A, when identical experiments were performed with the equally fluorescent doxorubicinol, intracellular fluorescence was even weaker for $\mathrm{MCF}-7_{\mathrm{DOX} 2-12}$ cells. This may reflect a reduced and greatly reduced ability of doxorubicinol to enter MCF$7_{\mathrm{CC} 12}$ and MCF-7 ${ }_{\text {DOX2-12 }}$ cells, respectively. When microscope settings were adjusted to improve detection of these weak signals (Figure 5B), it was clear that doxorubicinol, unlike doxorubicin, localized outside of the nucleus in both cell lines, suggesting that the metabolite cannot reach or bind its target. This raises the prospect that some of the extra nuclear doxorubicin in MCF-7 DOX2-12 cells may, in fact, be doxorubicinol or another fluorescent doxorubicin metabolite. However, the doxorubicin fluorescence in MCF-7 ${ }_{\text {DOX2-12 }}$ cells is much more concentrated in the perinuclear region and not as diffuse as doxorubicinol, suggesting the drug and its metabolite occupy distinct locations within cells.

We then assessed whether co-treatment of cells with $5 \beta$-cholanic acid altered doxorubicin or doxorubicinol localization (Figure 5). Interestingly, $200 \mu \mathrm{M} 5 \beta$-cholanic acid was able to completely restore doxorubicin localization to the nucleus of MCF- $7_{\text {DOX2-12 }}$ cells, suggesting that the conversion of doxorubicin to doxorubicinol does alter the drug's ability to reach or bind its target. The same concentration of $5 \beta$-cholanic acid, however, had no effect on doxorubicinol localization in MCF-7 ${ }_{\mathrm{CC} 12}$ and MCF-7 DOX2-12 $_{2}$ cells.

\section{Doxorubicinol fails to accumulate in MCF-7 $7_{\mathrm{CC} 12}$ and MCF-7 DOX2-12 $^{\text {cells }}$}

After incubation with $0.5 \mu \mathrm{M}$ doxorubicin, we used high performance liquid chromatography to assess the level

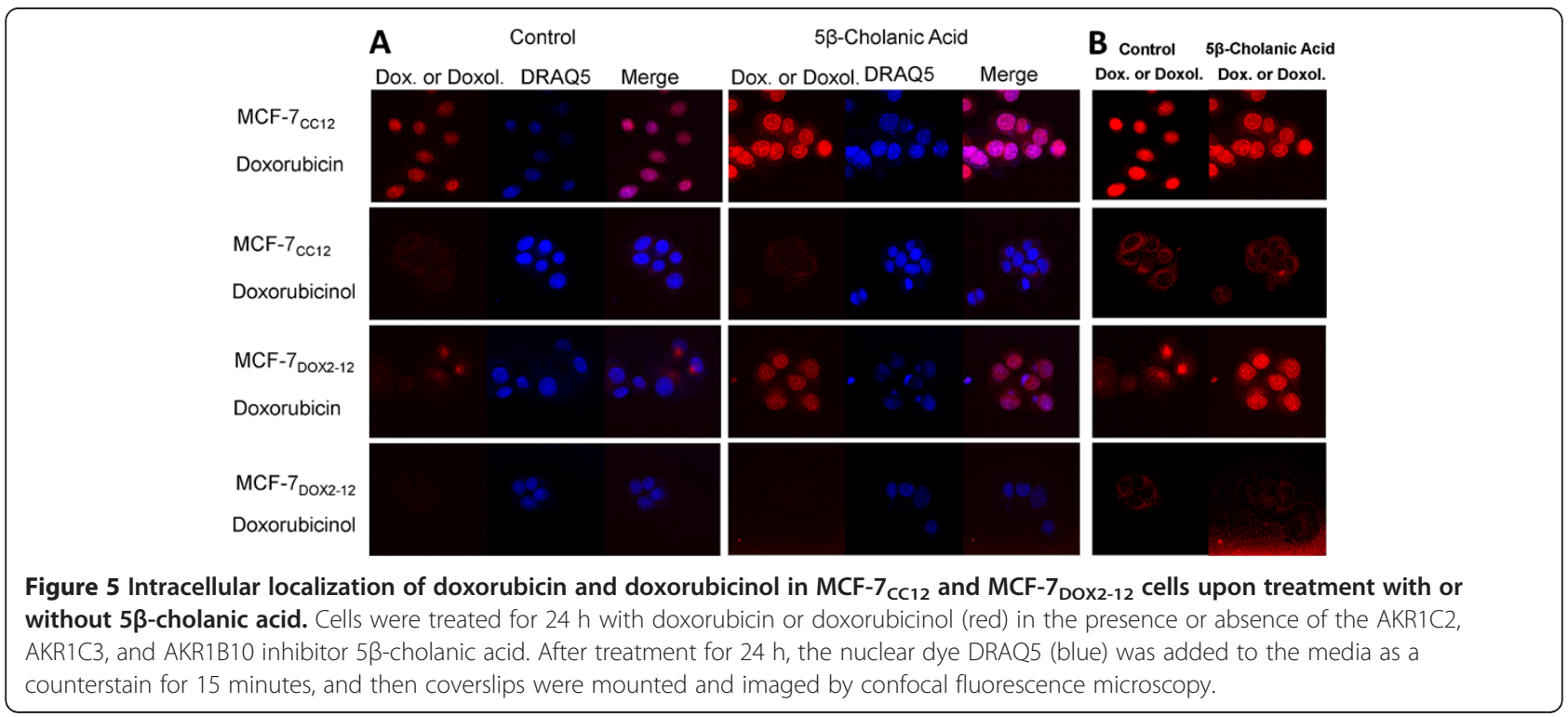


of doxorubicin and doxorubicinol in $\mathrm{MCF}-7_{\mathrm{CC} 12}$ and MCF-7 DOX2-12 cells and in the medium in which they grew. As shown in Figure 6, there was no detectable doxorubicinol in doxorubicin-treated $\mathrm{MCF}-7_{\mathrm{CC} 12}$ cells or in their cell culture medium, suggesting minimal expression of AKRs or CBRs. However, we surprisingly did not detect any doxorubicinol in MCF-7 DOX2-12 $_{\text {cells or }}$ their medium, despite their higher levels of expression

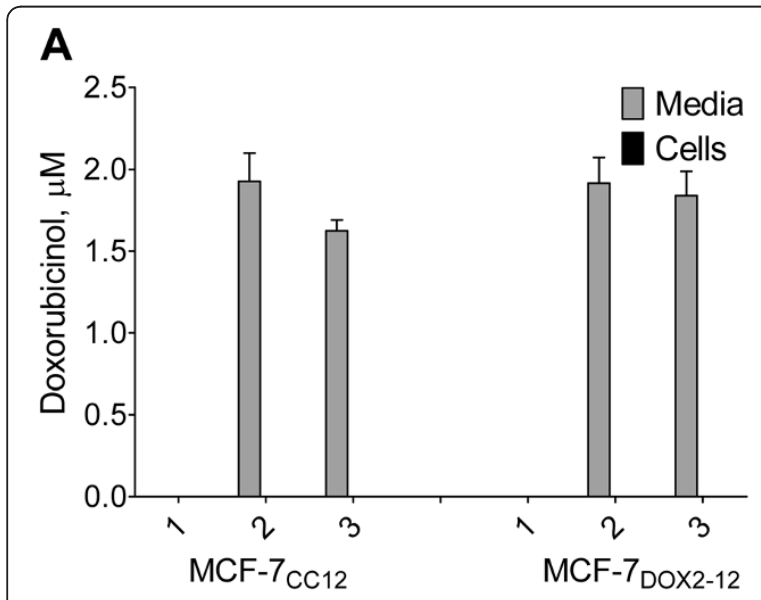

B

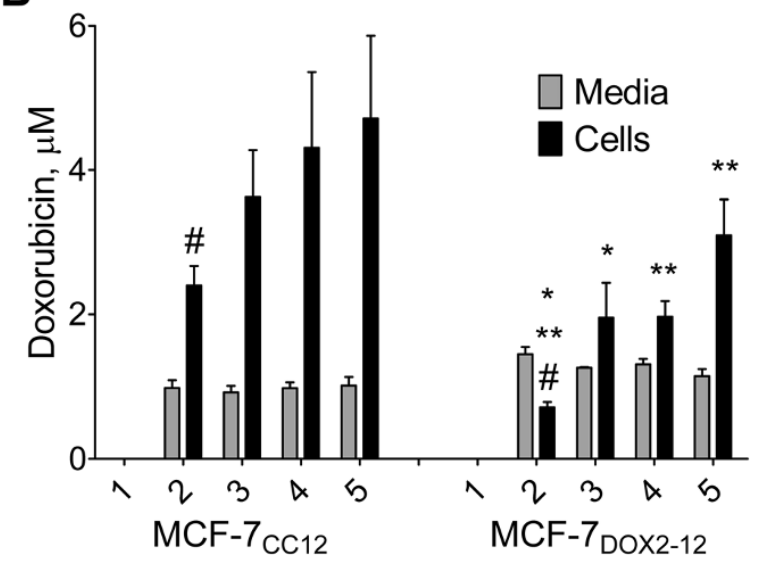

Figure 6 Intracellular levels of doxorubicin or doxorubicinol in the presence or absence of $5 \beta$-cholanic acid or cyclosporine $A$, measured by HPLC. MCF-7 ${ }_{C C 12}$ and MCF-7DOX2-12 cells were treated for $24 \mathrm{~h}$ with $0.5 \mathrm{uM}$ doxorubicin or doxorubicinol, 200uM $5 \beta$-cholanic acid, and/or 5 uM cyclosporine A. After treatment, cellular and media extracts were prepared and assessed for doxorubicin or doxorubicinol content by HPLC: (A) Treatment with doxorubicinol and $5 \beta$-cholanic acid: $1=$ no treatment; $2=0.5 \mathrm{uM}$ doxorubicinol; $3=0.5 \mathrm{uM}$ doxorubicinol and 200uM 5 3 -cholanic acid, (B) Treatment with doxorubicin, $5 \beta$-cholanic acid, and/or cyclosporine $\mathrm{A}: 1=$ no treatment; $2=0.5 \mathrm{uM}$ doxorubicin; $3=0.5 \mathrm{uM}$ doxorubicin $+200 \mathrm{uM} 5 \beta$-cholanic acid; $4=0.5 \mathrm{uM}$ doxorubicin $+5 \mathrm{uM}$ cyclosporine $\mathrm{A} ; 5=0.5 \mathrm{uM}$ doxorubicin $+200 \mathrm{uM} 5 \beta$-cholanic acid +5uM cyclosporine A. The symbol \# represents differences between MCF-7 CC12 $_{2}$ and MCF-7Dox2-12 cells (treatment 2) with a $p$ value $\leq 0.01$. The symbols ${ }^{*}$ and ${ }^{* *}$ represent significant differences between treatments at $p$ values of $\leq 0.05$ and $\leq 0.01$, respectively. of AKR isoforms in the cells. Added doxorubicinol to cells could be extracted and quantified in the medium and in cells (Figure 6A), suggesting that the negative result was not due to an inability of the method to detect doxorubicinol. Treatment of either cell line with $5 \beta$ cholanic acid did not affect the intracellular level of doxorubicinol or the levels of doxorubicinol in the media.

Intracellular levels of doxorubicin are significantly altered upon treatment of MCF-7 $7_{\text {DOX2-12 }}$ cells with $5 \beta$-cholanic acid and/or cyclosporine $A$

Treatment of $\mathrm{MCF}-7_{\mathrm{CC} 12}$ cells with $5 \beta$-cholanic acid and the pan $\mathrm{ABC}$ transporter inhibitor cyclosporine $\mathrm{A}$ increased cellular doxorubicin content by $51 \%$ and $80 \%$, respectively (Figure 6B). Addition of both agents increased doxorubicin content to almost twice that of untreated cells, but none of the above differences in doxorubicin content were considered statistically significant. In contrast, $5 \beta$-cholanic acid or cyclosporine A significantly increased doxorubicin content in MCF-7 ${ }_{\text {DOX2-12 }}$ cells by 2.8-fold (Figure 6B). Treatment of MCF-7 DOX2-12 cells with both $5 \beta$-cholanic acid and cyclosporine $\mathrm{A}$ increased cellular doxorubicin content to levels 4.4-fold higher than untreated cells (Figure 6B). These differences relative to untreated cells were found to be highly significant, and are likely due to the increased expression of AKRs [17] and $A B C$ drug transporters known to be overexpressed in MCF-7 ${ }_{\text {DOX2-12 }}$ cells, including Abcc1 [27].

\section{Doxorubicinol binds to DNA with lower affinity than doxorubicin}

We theorized that doxorubicinol does not localize to the nuclei of $\mathrm{MCF}-7_{\mathrm{CC} 12}$ and $\mathrm{MCF}-7_{\mathrm{DOX} 2-12}$ cells because the hydroxylation of doxorubicin reduces its affinity for DNA. To test this hypothesis, we compared the DNA binding parameters of doxorubicin and doxorubicinol using a binding displacement assay described in Methods. As shown in Figure 7 and Additional file 3: Table S3, both $B_{\max }$ and $K_{\text {app }}$ were substantially different between doxorubicinol $(0.667 \pm 0.013$ and $0.679 \pm$ $0.034 \mu \mathrm{M}$, respectively) and doxorubicin $(0.903 \pm 0.012$ and $0.412 \pm 0.017 \mu \mathrm{M}$, respectively), suggesting that, on a molar basis, doxorubicinol binds to DNA with a much lower affinity and capacity than doxorubicin.

\section{Discussion}

Use of the binomial statistic to interpret the significance of pathways in gene expression data

DNA microarray, high throughput quantitative PCR, and other gene profiling approaches have been highly useful in identifying differences in gene expression between cells or tumours responding to chemotherapy 


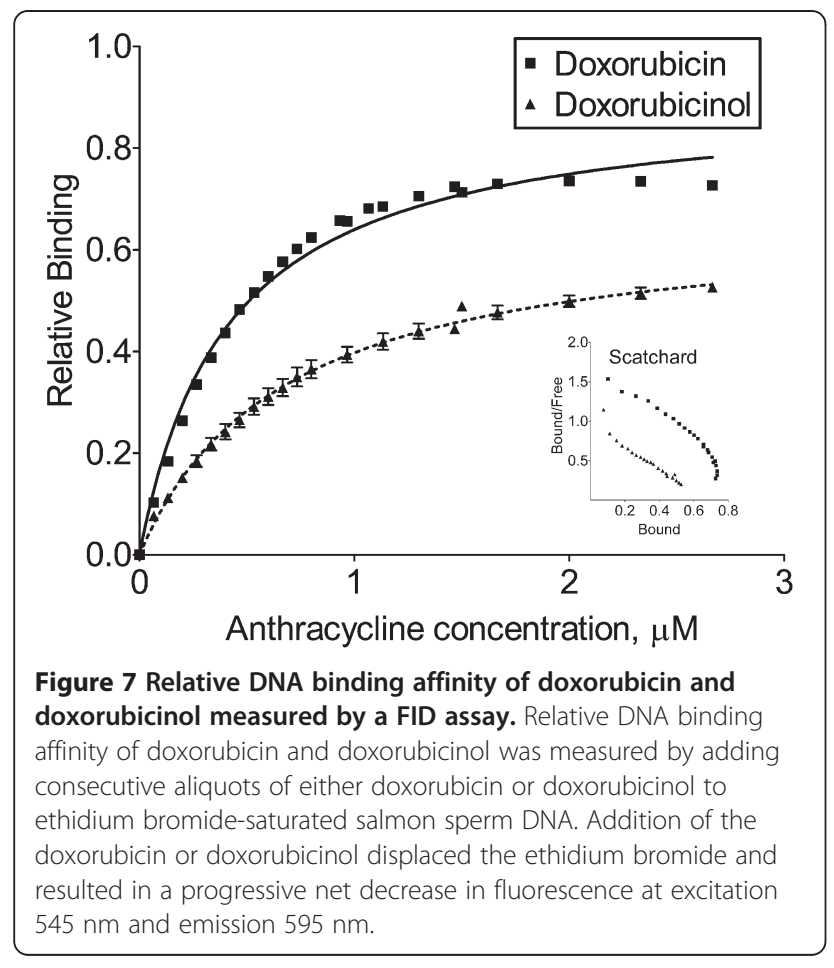

agents and those that do not. Unfortunately, the false discovery rate for such approaches is quite high, largely due to the identification of a large number of "passenger genes" unrelated to drug response. A wide variety of pathway analysis tools exist today, some manually curated, and some created primarily through machine learning. PharmGKB [28], Ariadne Pathway Studio [29], Reactome [30], Ingenuity Pathway Analysis (http://www. ingenuity.com/), GenMAPP [31], and DAVID [32], are examples of available tools which can be used to map changes in gene expression to alterations in biochemical pathways. The difficulty with this approach is the sheer size of the data sets, the large number of documented pathways, and the complex statistics required to determine the significance of findings. In this study we elected to use a simple model to examine the biology of doxorubicin resistance, namely looking for "overrepresentation" of doxorubicin pharmacokinetic and pharmacodynamic genes in datasets of genes having altered expression in doxorubicin resistance.

In order to assess the feasibility of this approach and to survey the broadest number of genes, we used Agilent full genome microarrays containing 27,958 Entrez gene probes, unlike our previous study of only 1720 gene probes [17]. This approach helped to uncover a number of AKRs induced during selection for doxorubicin resistance, including AKR1C1, AKR1B1, AKR1B10, and AKR1C3. Their expression was elevated between 4.5and 13.4-fold (Additional file 1: Table S1). Given that the probes for these AKRs on the Agilent microarrays were not isoform-specific, we used RTqPCR with isoformspecific primers (Table 4) to determine that, upon selection for doxorubicin resistance, transcripts for AKR1C2, AKR1C3, and AKR1B10 were overexpressed 3.6-, 9.1-, and 10.4-fold, respectively (Figure 3 ). In addition to the AKRs, other over-represented genes (Figure 2) provide further insight into other proteins that likely contribute to doxorubicin resistance. For example, NQO1 codes for $\mathrm{NAD}(\mathrm{P}) \mathrm{H}$ dehydrogenase quinone 1 , which plays a role in converting doxorubicin to doxorubicin deoxyaglycone or to doxorubicin semiquinone (Figure 2). Its 3-fold increase in expression might therefore increase the conversion of doxorubicin to these metabolites as well. Transcripts for the drug efflux pump Abcc1 were also upregulated 8.3-fold, as well as transcripts for other ATP-binding cassette $(\mathrm{ABC})$ transporters such as Abcd3, Abcg2, and Abca1. In addition, a gene (SLC22A15) homologous to the solute carrier protein Slc22a16 (which promotes doxorubicin uptake into cells [33]) was found to be down regulated by 2.8 -fold. The combined changes in the expression of $\mathrm{ABC}$ transporters and solute carrier proteins would be expected to reduce doxorubicin accumulation into cells. The gene for catalase $(C A T)$ was found to be upregulated 3.6-fold in MCF-7 ${ }_{\text {DOX2-12 }}$ cells. Since its gene product helps protect cells from oxidative damage by reactive oxygen species [34], its elevated expression would protect cells from reactive oxygen species known to be generated by doxorubicin. Genes associated with the cardiotoxicity of doxorubicin (through negative effects on mitochondrial function when converted to doxorubicinol) also have altered expression in breast tumour cells upon selection for doxorubicin resistance, including ACO1, ATPS, CYCS, and ATP2B4 (Figure 2 and Additional file 1: Table S1).

Of the above-described changes in gene expression, the greatest were for the AKRs. Evidence provided in this study supports their substantial role in doxorubicin resistance in tumour cells in vitro, and possibly in the tumours of cancer patients. While many of the changes in gene expression identified in our microarray study likely play a bona fide role in doxorubicin resistance (given their roles in cells), some of the identified genes may not be the "drivers" of drug resistance, but change expression through the altered expression of the driver genes.

\section{Role of the AKRs in resistance to doxorubicin}

A role for AKRs in xenobiotic and anthracycline metabolism has already been well established in the literature [21,35-37]. We also published previously that aldo-keto reductases (AKRs) are overexpressed upon acquisition of anthracycline resistance, that doxorubicin localization to the nucleus is altered in doxorubicin-resistant cells, and that inhibition of AKRs restores doxorubicin sensitivity 
in doxorubicin-resistant cells [17]. However, the current study significantly extends these observations in many respects. For example, it reveals that the expression of other members of the AKR family is elevated as breast tumour cells acquire resistance to doxorubicin. This would further increase the production of doxorubicinol and its possible conversion to other downstream metabolites. Moreover, our study provides a detailed comparison between doxorubicin and doxorubicinol in terms of their cytotoxicity, subcellular localization, and DNA binding activity.

Interestingly, despite having identical fluorescence capacities, cellular levels of doxorubicinol in both MCF$7_{\mathrm{CC} 12}$ and MCF-7 $7_{\mathrm{DOX} 2-12}$ cells was considerably lower than that of doxorubicin (as measured by cellular fluorescence intensity after drug administration and washing away free drug not taken up by cells). This decreased doxorubicinol uptake may be because hydroxylated doxorubicin is more polar and less able to traverse the hydrophobic plasma membrane. Moreover, even if the confocal microscope settings are modified to allow greater sensitivity to detect cellular doxorubicinol, doxorubicinol was found not to be localized to the nucleus in both MCF-7 $7_{\mathrm{CC} 12}$ and MCF-7 $7_{\mathrm{DOX} 2-12}$ cells. This indicates that the differential localization of doxorubicin between $\mathrm{MCF}-7_{\mathrm{CC} 12}$ and MCF-7 ${ }_{\text {DOX2-12 }}$ cells may be due to the strongly elevated conversion of doxorubicin to doxorubicinol (or other fluorescent metabolites) in MCF-7 $7_{\text {DOX2-12 }}$ cells. This may be why "doxorubicin" had an altered location in anthracycline-resistant cells in our previous study. The fluorescence observed in lysosomes may be that of doxorubicin, but also of doxorubicinol and other fluorescent doxorubicin metabolites. Consistent with this view, and not reported in our previous study, the administration of the AKR inhibitor $5 \beta$-cholanic acid significantly restored "doxorubicin" localization to the nucleus. More likely the inhibitor prevented doxorubicin conversion to doxorubicinol, permitting more doxorubicin to be retained within the nucleus.

What could account for the decreased localization of doxorubicin to the nucleus? We report in the current study that doxorubicinol has significantly lower ability to bind to DNA than doxorubicin (altered $\mathrm{B}_{\max }$ and $\mathrm{K}_{\text {app }}$ ). The conversion of doxorubicin to doxorubicinol by AKRs would result in reduced binding to DNA and hence less ability of the drug to remain associated with the nucleus. In our previous study, we did not differentiate between the cellular localization of doxorubicin and doxorubicinol.

One surprising finding in our study was the lack of detection of significant doxorubicinol in MCF-7 $7_{\text {DOX2-12 }}$ cells (Figure 6). This was despite the elevated expression of a number of AKRs in the cell line (Figure 3), which would be expected to covert doxorubicin to doxorubicinol. And yet, the addition of $5 \beta$-cholanic acid with doxorubicin increased the cellular content of doxorubicin (Figure 6), supporting the observation that $5 \beta$-cholanic acid is able to block the conversion of doxorubicin to doxorubicinol. What may account for the discrepancy in these points of view? One possibility is that $5 \beta$-cholanic acid blocks the efflux of doxorubicin by drug transporters (possibly Abcc1), thereby increasing the retention of doxorubicin in cells. One argument against this hypothesis is that both $5 \beta$-cholanic acid and cyclosporine A increased cellular doxorubicin content (Figure 6), the latter being a known inhibitor of Abcc1 function [38]. The combination of both agents increased cellular doxorubicin content further, suggesting that they were acting by distinct mechanisms. Moreover, unlike $5 \beta$-cholanic acid (Figure 4), addition of cyclosporine A had no effect on the cytotoxicity of doxorubicin in

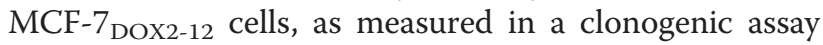
[27]. Finally, another inhibitor of AKR catalytic activity with a structure very distinct from cyclosporine A (flufenamic acid) also restored doxorubicin cytotoxicity and nuclear localization in MCF-7 DOX2-12 $_{\text {cells (data not }}$ shown). This suggests that it is the ability of these agents to inhibit AKR activity that is responsible for the restoration of drug cytotoxicity. An alternative argument is that the doxorubicinol, once formed, is further metabolized, such that the metabolite is not retained in the method used to extract cellular doxorubicin and doxorubicinol for HPLC-based measurements. Thus, doxorubicinol would not be seen to accumulate in MCF-7 ${ }_{\text {DOX2-12 }}$ cells.

Despite the ability of both cyclosporin A and $5 \beta$ cholanic acid to increase cellular doxorubicin content in MCF-7 DOX2-12 cells, why was only the latter agent able to appreciably restore doxorubicin cytotoxicity? Increasing the cellular content of doxorubicin by the cyclosporinemediated reduction of drug efflux may not sufficiently increase its cytotoxicity if the additional cellular doxorubicin is rapidly converted to doxorubicinol by the elevated expression of AKRs and/or if the additional doxorubicin is sequestered into lysosomes. In contrast, AKR inhibition may block all conversion of doxorubicin to doxorubicinol, such that any drug entering the cell remains as doxorubicin and is able to rapidly reach the nucleus, before being sequestered.

\section{Conclusions}

Using a full genome approach, this study provides important new insight into pharmacokinetic and pharmacodynamic pathways that are altered upon selection of cells for resistance to doxorubicin. In addition to our previously reported finding of increased expression of the AKR 1C isoforms [17], the current study reveals other changes in gene expression that would be expected 
to affect the cytotoxicity of doxorubicin. This includes genes that may: decrease uptake of doxorubicin (SLC22A15), enhance efflux of doxorubicin (ABCC1, ABCG2, ABCD3, ABCA1), enhance conversion of doxorubicin to doxorubicinol (AKR1B10, AKR1B1), doxorubicin deoxyaglycone or doxorubicin semiquinone (NQO1), and inhibit the ability of doxorubicin to damage tumour cells through the generation of reactive oxygen species (CAT). Moreover, this study provides an in-depth comparison of the biochemical properties of doxorubicin versus doxorubicinol. While the former is highly cytotoxic, has high DNA binding affinity, and localizes to the nucleus in wildtype breast tumour cells, doxorubicinol is over a million times less cytotoxoic, has significantly reduced DNA binding activity, and is retained in the cytoplasm or lysosomes of cells. We also show that the administration of AKR inhibitors with doxorubicin in MCF-7 DOX2 $_{2}$ cells substantially restores both drug localization to the nucleus and drug cytotoxicity. Interestingly, doxorubicinol is highly cardiotoxic, and it is believed that doxorubicinol is responsible for the cardiotoxicity associated with doxorubicin chemotherapy $[39,40]$. Since the AKR inhibitor $5 \beta$-cholanic acid is a well-tolerated naturally occurring bile acid in humans, and since flufenamic acid has been used in clinical trials with manageable toxicities [41], there may be significant value in conducting clinical trials in which either $5 \beta$-cholanic acid or flufenamic acid are coadministered with doxorubicin during chemotherapy. Results in this study would suggest that these AKR inhibitors may increase tumour levels of doxorubicin and block cardiotoxicity induced by doxorubicin conversion to doxorubicinol. This may dramatically improve the therapeutic index of doxorubicin when administered to cancer patients and improve the duration of clinical response for this otherwise highly effective chemotherapy drug.

\section{Methods}

\section{Supplies and reagents}

Supplies and reagents used in this study came from a variety of sources. Unless otherwise noted, Sigma (St. Louis, MO) was the supplier.

\section{Cell culture}

MCF-7 breast adenocarcinoma cells were obtained from the American Tissue Culture Collection (ATCC) (lot HTB-22) and selected for resistance to doxorubicin (Pfizer Pharmaceuticals, St. Laurent, QC) as previously described [27]. Briefly, doxorubicin-sensitive, wildtype MCF-7 cells were grown in progressively increasing concentrations of doxorubicin from $1000 x$ below the $\mathrm{IC}_{50}$ for the drug in parental MCF-7 cells (dose 1) to its maximally tolerated dose (dose 12) in 1.5- or 3-fold increments, with retention of cells surviving the greater of the two doses. Cells selected for survival in the varying doses of doxorubicin were termed MCF-7 $7_{\mathrm{DOX} 2}$ cells. A "co-cultured control" cell line was selected under identical conditions in the absence of drug $\left(\mathrm{MCF}-7_{\mathrm{CC}}\right.$ cells). These cells served as a control to help identify changes in gene expression due to long-term cell culture. The highest dose level to which cells were selected are indicated in the subscript of the cell line name. For example, MCF-7 ${ }_{\text {DOX2-12 }}$ cells refers to cells selected to the $12^{\text {th }}$ dose level of doxorubicin. The 2 in the subscript is to prevent confusion with a previously isolated doxorubicin-resistant cell line in our laboratory (MCF$\left.7_{\text {DOX }}\right)$. All cells used in this study were selected to dose level 12 (0.1 $\mu \mathrm{M}$ doxorubicin). Cells were grown in highglucose DMEM medium (Fisher Scientific, Nepean, ON) supplemented with penicillin-streptomycin (Fisher) and $10 \%$ fetal bovine serum (Fisher) in $75 \mathrm{~cm}^{2}$ tissue culture flasks (Sarstedt Canada, Montreal, QC), unless otherwise noted. Cells were maintained at $37^{\circ} \mathrm{C}$ in air supplemented with $5 \% \mathrm{CO}_{2}$ in a humidified environment. Cells were passaged weekly, with a medium change once between passages. Drug-resistant cells were maintained in medium containing doxorubicin at their selection dose.

\section{Microarray analysis}

Changes in gene expression between $\mathrm{MCF}-7_{\mathrm{CC} 12}$ and MCF-7 DOX2-12 $_{\text {cells }}$ were identified by microarray analysis using Agilent $4 \times 44 \mathrm{k}$ whole human genome arrays (product number G4112F; Agilent Technologies, Mississauga, $\mathrm{ON}$ ). These arrays enabled us to determine the level of expression of 27,958 human Entrez genes (close to the entire genome). Five hundred ng of total RNA, isolated with a Qiagen RNeasy kit (Mississauga, ON), was used for each sample. The RNA was then labeled with Cy3 or Cy5 using an Agilent Quick Amp labeling kit (Product \#5190-0444). Hybridization was performed as per the manufacturer's protocol. Experiments were repeated using multiple batches of labeled RNA, with both forward and reverse-labeling to account for dye bias, for a total of 16 two-colour arrays. The microarrays were scanned, and feature extraction and background intensity corrections were performed with Agilent software (v.10.7.3.1). Using Partek Genomics suite (St. Louis, MO) to perform a 4 way ANOVA using the Method of Moments [42], a list of genes significantly over- or underexpressed in MCF-7 $7_{\text {DOX2-12 }}$ cells relative to $\mathrm{MCF}-7_{\mathrm{CC} 12}$ cells. The false discovery rate was set at 0.01 , with only genes changing expression by $\geq 2$-fold being noted. The four variables assessed in the 4 way ANOVA were the cell line (MCF-7 $7_{\mathrm{CC} 12}$ cells versus MCF-7 ${ }_{\mathrm{DOX} 2-12}$ cells), the dye used (Cy3 versus Cy5), the experimental batch of arrays (to address batch effects) and the arrays themselves to address random effects. The input file was the 
data from all 16 two-colour arrays comparing gene expression between MCF-7 ${ }_{\text {DOX2-12 }}$ and MCF-7 ${ }_{\mathrm{CC} 12}$ cells. The model used was: Yijklm $=\mu+$ Cell line $(i)+$ Dye $(j)+$ Exp batch $(\mathrm{k})+$ arrays (random effect) $(\mathrm{kl})+$ eijklm, where Yijklm represents the mth observation on the ith Cell line jth Dye kth Exp batch lth arrays, $\mu$ is the common effect for the whole experiment, eijklm represents the random error present in the mth observation, on the ith Cell line, jth Dye, kth Exp batch, lth arrays. The errors eijklm were assumed to be normally and independently distributed, with mean 0 and standard deviation $\delta$ for all measurements. Arrays and Exp batch were considered random effects. Normalized expression was transformed to the base 2.0, with $\mathrm{p}$ values reported for significance of differences in the expression of each gene. The output of the analysis was a $\mathrm{p}$ value for the significance of the observed fold change in expression of a particular gene and for the mean ratio of expression of a particular gene between the two cell lines. The microarray data was deposited in the NCBI Gene Expression Omnibus (GEO) database, accession number GSE27254) in accordance with MIAME standards [15]. The url to access this data is: http://www.ncbi.nlm.nih.gov/geo/query/acc.cgi?token= dbezngycywquuhm\&acc=GSE27254.

To confirm the isogenic nature of the two cell lines, a Significance Analysis of Microarrays (SAM) plot was created using the freely-available microarray software TM4 suite [43], with the following workflow:

(1) Agilent raw data was converted to .MEV format using TIGR Express Converter v2.1.

(2) The MEV files were then normalized using TIGR MIDAS v2.22 and a LOWESS normalization filter with flip-dye consistency checking where appropriate.

(3) A one-class Significance Analysis of Microarrays (SAM) analysis was performed using TIGR MEV v4.6.1.

For pathway analysis, the list of significant differentiallyexpressed genes generated by Partek Genomics Suite was compared to a curated list of genes, transcripts or proteins shown to be involved in doxorubicin pharmacokinetics and pharmacodynamics in tumour cells or cardiomyocytes, available from the Pharmacogenomics Knowledge Base (PharmGKB) [16,28]. These lists were then compared in S-Plus (v8.0) using the binomial statistical test as previously described [14].

\section{RNA extraction, reverse transcription, and quantitative polymerase chain reaction}

Given that the $1 \mathrm{C}$ and $1 \mathrm{~B} \mathrm{AKR}$ isoforms are highly conserved and that the probes for the $1 \mathrm{C}$ and $1 \mathrm{~B}$ AKR transcripts on the Agilent $4 \times 44$ microarrays were not isoform-specific, we designed isoform-specific $1 C$ and $1 \mathrm{~B}$ primers to accurately quantify the levels of expression of the various $1 \mathrm{C}$ and $1 \mathrm{~B}$ transcripts. These primers (Table 4) and isoform-specific primers for the carbonyl reductases (which also convert doxorubicin to doxorubicinol) were used in RTqPCR experiments. Total RNA was extracted from the MCF-7 ${ }_{\mathrm{CC} 12}$ and MCF-7 $\mathrm{DOX} 2-12$ cell lines using a Qiagen RNeasy kit (Mississauga, ON), reverse transcribed, and the cDNAs amplified using an ABI 7900HT quantitative PCR machine and SYBR Green 1 detection chemistry as described previously [44]. After RNA extraction and before reverse transcription, RNA was quantified and quality ensured using an Agilent Bioanalyzer 2100 RNA nano kit. $2 \mu \mathrm{g}$ of RNA was then DNase I (Invitrogen) treated. For reverse transcription, either MMLV reverse transcriptase (Invitrogen, Burlington, $\mathrm{ON}$ ) and an oligo- $\mathrm{dT}_{20}$ primer, or Superscript III reverse transcriptase (Invitrogen) and an AKR1C-specific reverse transcription primer were used, all according to the manufacturer's protocol. The cDNA was then stored at $-20^{\circ} \mathrm{C}$ until analysis via the $\triangle \mathrm{CT}$ method. All experiments were performed according to MIQE standards [45]. Primers used for reverse transcription or to amplify specific cDNAs are described in Additional file 4: Table S4.

\section{Protein extraction and quantification}

Ten $\mathrm{cm}$ plates of cells (cultured for 2 passages without drug to $80 \%$ confluence) were rinsed twice in Dulbecco's PBS, removed of excess liquid using a pipette tip, and placed on ice. Three hundred to $1000 \mu \mathrm{l}$ of RIPA buffer (10 mM Tris- $\mathrm{HCl}, 1 \%$ sodium deoxycholate, 0.1\% SDS, $1 \%$ Triton X-100, $150 \mathrm{mM} \mathrm{NaCl}, \mathrm{pH} 7.5)$ with added Complete $^{\mathrm{Ts}}$ protease inhibitors (Roche Diagnostics, Laval, QC) were added to each plate, and the plates scraped with a cell scraper. The resulting crude lysate was passed through a 21 gauge needle 5 times. The lysate was incubated on ice for 30 minutes and then centrifuged for 20 minutes at $13.2 \times 10^{3} \times g$ at $4^{\circ} \mathrm{C}$. The supernatant was retained and stored at $-80^{\circ} \mathrm{C}$. Protein concentrations in extracts were measured using a BCA protein quantification kit (Pierce Bioscience) using standard solutions of Bovine Serum Albumin (BSA).

\section{Western blotting}

Forty $\mu \mathrm{g}$ of protein were diluted in $6 \mathrm{x}$ Laemelli loading buffer and loaded onto a $10 \%$ SDS-PAGE gel with a $4 \%$ stacking gel. The gel was also loaded with $5 \mu \mathrm{L}$ of BioRad (Mississagua, ON) dual colour protein marker. The gels were subjected to electrophoresis in a Bio-Rad mini tetra system at $80 \mathrm{~V}$ for 30 minutes and then at $120 \mathrm{~V}$ for an additional $1 \mathrm{~h}$. The gels were removed and the gel proteins transferred to nitrocellulose membranes (GE Healthcare, Baie d'Urfe QC) using a Bio-Rad semi-dry 
electroblotting apparatus for $1 \mathrm{~h}$ at $12 \mathrm{v}$. Membranes were then stained with $0.5 \%$ Ponceau $\mathrm{S}$ in $1 \%$ acetic acid to confirm transfer efficiency and even protein loading. The membranes were blocked in $5 \%$ skim milk powder (Carnation) in $0.1 \%$ TBST $(20 \mathrm{mM}$ Tris- $\mathrm{HCl}, 150 \mathrm{mM}$ $\mathrm{NaCl}, 0.05 \%$ Tween 20, pH 7.5) for $1 \mathrm{~h}$ at room temperature, and then incubated overnight at $4^{\circ} \mathrm{C}$ with an isoform specific mouse monoclonal anti-AKR1C3 antibody [46] (Sigma) at a 1:2,000 dilution in 5\% skim milk powder in TBST. The membranes were then washed with TBST for 15 minutes, and incubated in HRP-conjugated goat anti-mouse secondary antibody (Santa Cruz Biotechnology Inc., Santa Cruz, CA) at a $1: 10,000$ dilution in $5 \%$ skim milk powder in TBST for $1 \mathrm{~h}$ at room temperature. Membranes were again washed in TBST for 15 minutes and subjected to $3 \times 5$ minute final washes in TBS before being covered in ECL solution (Santa Cruz Biotechnology Inc.) and imaged using a gel documentation system (Alpha Innotech) for 10 minutes. An identical procedure using a mouse monoclonal anti- $\beta$-tubulin antibody (Santa Cruz Biotechnology Inc.) at a 1:10,000 dilution was used to monitor $\beta$-tubulin levels in the extracts as a loading control.

\section{Confocal microscopy}

Cells were plated on \#1 coverslips placed in 6-well plates and allowed to adhere overnight before being treated. Treatment consisted of addition of $0.5 \mu \mathrm{M}$ doxorubicin or doxorubicinol (Toronto Research Chemicals Inc., North York, ON), and either $200 \mu \mathrm{M} 5 \beta$-cholanic acid (Steraloids, Newport, RI) or DMSO as a vehicle control. After the $24 \mathrm{~h}$ treatment, DRAQ5 (Biostatus, Leicestershire, UK) was added to the culture media for 15 minutes as a nuclear counterstain. The coverslips were rinsed gently in 3 sequential PBS washes and sealed onto standard microscope slides using clear nail polish. After the nail polish dried, cells were observed using a Zeiss LSM 510 META confocal laser scanning microscope using an argon-ion laser at a $488 \mathrm{~nm}$ wavelength band for excitation of doxorubicin and doxorubicinol and using a $560 \mathrm{~nm}$ long-pass filter to detect intrinsic fluorescence of doxorubicin and its metabolites. A $633 \mathrm{~nm}$ laser with a $650 \mathrm{~nm}$ long-pass filter was used to detect DRAQ5 fluorescence.

\section{High-performance liquid chromatography}

Cells $\left(8.0 \times 10^{6}\right.$ cells per $10 \mathrm{~cm}$ plate $)$ were allowed to adhere overnight, after which they were treated with $0.5 \mu \mathrm{M}$ doxorubicin or $0.5 \mu \mathrm{M}$ doxorubicinol (with or without $5 \mu \mathrm{M}$ cyclosporine $\mathrm{A}$ and/or $200 \mu \mathrm{M} 5 \beta$ cholanic acid) for $24 \mathrm{~h}$. After this time period, the media was decanted (with $0.5 \mathrm{~mL}$ reserved for HPLC analysis), and the plates were rinsed twice in PBS. One $\mathrm{mL}$ of a
$0.2 \mathrm{M} \mathrm{Na} \mathrm{HPO}_{4}$ solution, $\mathrm{pH}$ 8.5, was added to the plates and the cells were scraped off of the plate. A $0.5 \mathrm{ml}$ volume of the same solution was added to the $0.5 \mathrm{~mL}$ of reserved media. Each sample was then added to $4 \mathrm{~mL}$ of a 9:1 v/v chloroform:n-heptanol mixture in a polypropylene $15 \mathrm{~mL}$ centrifuge tube and shaken on a mixer for 20 minutes, after which the samples were centrifuged for 10 minutes at $2000 \times g$ at $20^{\circ} \mathrm{C}$. The bottom organic layer was then aspirated from the tube using a glass $5 \mathrm{~mL}$ pipette and dispensed into a new $15 \mathrm{~mL}$ centrifuge tube containing $250 \mathrm{uL}$ of $0.1 \mathrm{M}$ orthophosphoric acid. Each tube was then mixed on a vortex mixer for 30 seconds before being centrifuged for 2 minutes at $2000 \times g$. The top $200 \mu \mathrm{L}$ of the upper aqueous layer was then removed and stored at -80 degrees Celsius for later analysis.

Separations were performed using a revised gradient elution based on a previously described isocratic method [17] on a Waters Alliance e2695 system with a Waters 2475 fluorescence detector set at $480 \mathrm{~nm}$ excitation and $560 \mathrm{~nm}$ emission. Chromatographic conditions were the following: column: YMC CN $25 \times 5 \mathrm{~mm}$ column; Eluent A: $10 \mathrm{mM} \mathrm{NaH} \mathrm{PO}_{4} \mathrm{pH}$ 4.0, Eluent B: HPLC grade $\mathrm{CH}_{3} \mathrm{CN}$; flow rate: $1.0 \mathrm{~mL} / \mathrm{min}$. The gradient program was as follows: $0 \mathrm{~min}=20 \% \mathrm{~B} 80 \% \mathrm{~A}, 10 \mathrm{~min}=50 \% \mathrm{~B}$ $50 \% \mathrm{~A}, 11$ to $24 \mathrm{~min}=20 \% \mathrm{~B} 80 \% \mathrm{~A}$. The slope for each gradient change was linear.

\section{DNA binding affinity assay}

The relative DNA binding affinity of doxorubicin and doxorubicinol was determined by using a fluorescent intercalator displacement assay [47]. Briefly, a quartz cuvette was filled with $3 \mathrm{~mL}$ of Tris buffer $(0.1 \mathrm{M}$ Tris, $0.1 \mathrm{M} \mathrm{NaCl}, \mathrm{pH}$ 8.0; approximating conditions inside the nucleus) to which $4.4 \mu \mathrm{M}$ ethidium bromide was added. A fluorescence reading (excitation: $545 \mathrm{~nm}$, emission: $590 \mathrm{~nm}$ ) was taken using a Perkin Elmer LS-50 fluorimeter; this constituted the baseline reading. Pre-sheared salmon sperm DNA (8.8 $\mu \mathrm{M}$ in base pairs) was then added to the cuvette, incubated for 5 minutes, and again the fluorescence was determined; this constituted the maximal or $100 \%$ reading. Aliquots of doxorubicin or doxorubicinol $(0.067 \mu \mathrm{M})$ were added to the cuvette, incubated for 5 minutes, and the corresponding reading recorded. The background reading was subtracted for each reading and then divided by the maximal reading to determine per cent of maximal binding. These data were fit to curves to determine $K_{\text {app }}$ and $B_{\max }$ values.

\section{Measurement of drug sensitivity}

Drug sensitivity was assessed using a variation [48] of the standard clonogenic assay [49]. Briefly, for each condition, $12 \times 25 \mathrm{~cm}^{2}$ flasks were plated with $2.5 \times 10^{5}$ cells and left to adhere overnight. The next day, each flask 
was treated with a different concentration of doxorubicin, decreasing in 3-fold increments, from $3.0 \times 10^{-6} \mathrm{M}$ to $5.13 \times 10^{-11} \mathrm{M}$, with a final flask receiving no doxorubicin. After $24 \mathrm{~h}$, cells were trypsinized, pelleted, and resuspended in $300 \mathrm{uL}$ of medium which was then combined with $2.7 \mathrm{~mL}$ of methyl cellulose growth medium [2.6\% methyl cellulose, (Shin-Etsu) and 30\% FBS in IMDM (Princess Margaret Hospital)]. After being mixed thoroughly, the suspension was allowed to settle for 30 minutes before $1.2 \mathrm{ml}$ of cells were introduced into 6-well tissue culture plates. Plates were incubated for 2 weeks and then 10 randomly selected fields in each well were counted at 40x magnification.

\section{Statistical analyses}

Graphpad Prism (v5.0) was used for all statistical tests unless otherwise noted. Differences between treatment means were assessed using either a Student's unpaired t-test or an unpaired 1-way Analysis of Variance (ANOVA) with Tukey's Honestly Significant Difference (HSD) posthoc test where appropriate. A p value $\leq 0.05$ was considered significant.

\section{Additional files}

Additional file 1: Table S1. Genes associated with doxorubicin

pharmacokinetics or pharmacodynamics in cancer cells or

cardiomyocytes as identified in the PharmGKB knowledgebase. Those genes identical to and related to genes significantly changing expression upon acquisition of doxorubicin resistance in MCF-7 breast tumour cells by microarray analysis (false discovery rate of 0.01 ) are listed in bold regular font and bold italics font, respectively. The fold change in gene expression is also listed for upregulated $(+)$ or down regulated $(-)$ genes.

Additional file 2: Table S2. Over-representation of doxorubicin pharmacokinetic or pharmacodynamic genes in the dataset of genes associated with the acquisition of doxorubicin resistance in MCF-7 breast tumour cells. The $p$ values assessing the significance of this overrepresentation are depicted in parentheses, with statistically significant $p$ values listed in bold font.

Additional file 3: Table S3. Differences in doxorubicin and doxorubicinol DNA binding parameters. DNA binding by doxorubicin or doxorubicinol was compared in an ethidium bromide displacement assay as described in Materials and Methods. The $B_{\max }$ and $K_{a p p}$ values for doxorubicin and doxorubicinol are listed, along with the $p$ values for significant differences in their binding parameters $(p<0.05)$.

Additional file 4: Table S4. Primers used for measurement of expression of candidate genes involved in doxorubicin hydroxylation by quantitative PCR. Forward and reverse primers recognizing aldo ketoreductases (AKRs) or carbonyl reductases (CRs) are listed, along with the primers for the reference gene RPS28.

\section{Abbreviations}

ABCC1: ATP-binding cassette protein C1; AKR: Aldo-keto reductase; ANOVA: Analysis of variance; $B_{\max }$ : Measure of the number of receptors for a particular drug; CBR: Carbonyl reductase; HPLC: High performance liquid chromatography; $\mathrm{IC}_{50}$ : Concentration required to inhibit growth by $50 \%$; $K_{\text {app }}$ : Apparent dissociation constant; MIAME: Minimum information associated with a microarray experiment; NQO1: NAD(P)H dehydrogenase, quinine 1; PCR: Polymerase chain reaction; PharmGKB: Pharmacogenomics Knowledgebase.

\section{Competing interests}

The authors declare that they have no competing interests.

\section{Authors' contributions}

$\mathrm{AH}$ participated in writing of the manuscript and performed clonogenic experiments, HPLC experiments, doxorubicin binding assays, and statistical tests. BG performed all microarray experiments and analyzed the microarray data. JS performed the doxorubicin and doxorubicinol localization experiments by confocal microscopy. DM supervised HPLC experiments, including optimization of detection of doxorubicin metabolites. AP devised and supervised the performance of the study, acquired grant funding to support the study, and helped write and revise the manuscript. He is also the corresponding author for this manuscript. All authors read and approved the final manuscript.

\section{Source of funding}

Canadian Institutes of Health Research (Grant MOP-8993 to A.M.P.)

\section{Acknowledgements}

This work was supported by a grant from the Canadian Institutes of Health Research (MOP-89993). Core funding to support this work is also acknowledged from the Northern Cancer Foundation.

\section{Author details}

${ }^{1}$ Graduate Program in Biology, Laurentian University, Sudbury, ON, Canada. ${ }^{2}$ Regional Cancer Program, Sudbury Regional Hospital, Sudbury, ON P3E 5J1, Canada. ${ }^{3}$ Graduate Program in Biomolecular Science, Laurentian University, Sudbury, ON, Canada. ${ }^{4}$ Division of Medical Sciences, Northern Ontario School of Medicine, Sudbury, ON, Canada. ${ }^{5}$ Divison Of Oncology, Faculty of Medicine, University of Ottawa, Ottawa, Canada.

Received: 19 January 2012 Accepted: 17 August 2012

Published: 31 August 2012

\section{References}

1. Schneider $Y$ J, Baurain $R$, Zenebergh A, Trouet A: DNA-binding parameters of daunorubicin and doxorubicin in the conditions used for studying the interaction of anthracycline-DNA complexes with cells in vitro. Cancer Chemother Pharmacol 1979, 2:7-10.

2. Foglesong PD, Reckord C, Swink S: Doxorubicin inhibits human DNA topoisomerase I. Cancer Chemother Pharmacol 1992, 30:123-125.

3. Lopez M: Anthracyclines in the adjuvant treatment of breast carcinoma: thirty years later. Clin Ter 2006, 157:165-177.

4. Kartner N, Riordan JR, Ling V: Cell surface P-glycoprotein associated with multidrug resistance in mammalian cell lines. Science 1983, 221:1285-1288.

5. Doyle LA, Ross DD: Multidrug resistance mediated by the breast cancer resistance protein BCRP (ABCG2). Oncogene 2003, 22:7340-7358.

6. Frank NY, Margaryan A, Huang Y, Schatton T, Waaga-Gasser AM, Gasser M, Sayegh MH, Sadee W, Frank MH: ABCB5-mediated doxorubicin transport and chemoresistance in human malignant melanoma. Cancer Res 2005, 65:4320-4333.

7. Borst P, Evers R, Kool M, Wijnholds J: A family of drug transporters: the multidrug resistance-associated proteins. J Natl Cancer Inst 2000, 92:1295-1302

8. Gavelova M, Hladikova J, Vildova L, Novotna R, Vondracek J, Krcmar P, Machala M, Skalova L: Reduction of doxorubicin and oracin and induction of carbonyl reductase in human breast carcinoma MCF-7 cells. Chem Biol Interact 2008, 176:9-18

9. Hurwitz SJ, Terashima M, Mizunuma N, Slapak CA: Vesicular anthracycline accumulation in doxorubicin-selected U-937 cells: participation of lysosomes. Blood 1997, 89:3745-3754

10. Rajagopal A, Simon SM: Subcellular localization and activity of multidrug resistance proteins. Mol Biol Cell 2003, 14:3389-3399.

11. Aas T, Borresen AL, Geisler S, Smith-Sorensen B, Johnsen H, Varhaug JE, Akslen LA, Lonning PE: Specific P53 mutations are associated with de novo resistance to doxorubicin in breast cancer patients. Nat Med 1996, 2:811-814

12. Fischer V, Einolf HJ, Cohen D: Efflux transporters and their clinical relevance. Mini Rev Med Chem 2005, 5:183-195. 
13. Leonard GD, Fojo T, Bates SE: The role of $A B C$ transporters in clinical practice. Oncologist 2003, 8:411-424.

14. Cho RJ, Campbell MJ: Transcription, genomes, function. Trends Genet 2000, 16:409-415.

15. Brazma A, Hingamp P, Quackenbush J, Sherlock G, Spellman P, Stoeckert C, Aach J, Ansorge W, Ball CA, Causton HC, Gaasterland T, Glenisson P, Holstege FC, Kim IF, Markowitz V, Matese JC, Parkinson H, Robinson A, Sarkans U, Schulze-Kremer S, Stewart J, Taylor R, Vilo J, Vingron M: Minimum information about a microarray experiment (MIAME)-toward standards for microarray data. Nat Genet 2001, 29:365-371.

16. Thorn CF, Oshiro C, Marsh S, Hernandez-Boussard T, McLeod H, Klein TE, Altman RB: Doxorubicin pathways: pharmacodynamics and adverse effects. Pharmacogenet Genomics 2011, 21:440-446.

17. Veitch ZW, Guo B, Hembruff SL, Bewick AJ, Heibein AD, Eng J, Cull S, Maclean DA, Parissenti AM: Induction of $1 C$ aldoketoreductases and other drug dose-dependent genes upon acquisition of anthracycline resistance. Pharmacogenet Genomics 2009, 19:477-488.

18. Minotti G, Menna P, Salvatorelli E, Cairo G, Gianni L: Anthracyclines: molecular advances and pharmacologic developments in antitumor activity and cardiotoxicity. Pharmacol Rev 2004, 56:185-229.

19. Penning TM, Drury JE: Human aldo-keto reductases: Function, gene regulation, and single nucleotide polymorphisms. Arch Biochem Biophys 2007, 464:241-250

20. Kassner N, Huse K, Martin HJ, Godtel-Armbrust U, Metzger A, Meineke I, Brockmoller J, Klein K, Zanger UM, Maser E, Wojnowski L: Carbonyl reductase 1 is a predominant doxorubicin reductase in the human liver. Drug Metab Dispos 2008, 36:2113-2120.

21. Novotna R, Wsol V, Xiong G, Maser E: Inactivation of the anticancer drugs doxorubicin and oracin by aldo-keto reductase (AKR) 1C3. Toxicol Lett 2008, 181:1-6.

22. Chang BK, Brenner DE, Gutman R: Cellular pharmacology of doxorubicinol alone and combined with verapamil in pancreatic cancer cell lines. Anticancer Res 1989, 9:341-345.

23. Bernardini N, Giannessi F, Bianchi F, Dolfi A, Lupetti M, Zaccaro L, Malvaldi $G$, Del TM: Comparative activity of doxorubicin and its major metabolite, doxorubicinol, on V79/AP4 fibroblasts: a morphofunctional study. Exp Mol Pathol 1991, 55:238-250.

24. Endo $S$, Matsunaga T, Mamiya H, Ohta C, Soda M, Kitade Y, Tajima K, Zhao HT, El-Kabbani O, Hara A: Kinetic studies of AKR1B10, human aldose reductase-like protein: endogenous substrates and inhibition by steroids. Arch Biochem Biophys 2009, 487:1-9.

25. Steckelbroeck S, Oyesanmi B, Jin Y, Lee SH, Kloosterboer HJ, Penning TM: Tibolone metabolism in human liver is catalyzed by 3alpha/3betahydroxysteroid dehydrogenase activities of the four isoforms of the aldo-keto reductase (AKR)1C subfamily. J Pharmacol Exp Ther 2006 , 316:1300-1309.

26. Coley HM, Amos WB, Twentyman PR, Workman P: Examination by laser scanning confocal fluorescence imaging microscopy of the subcellular localisation of anthracyclines in parent and multidrug resistant cell lines. Br J Cancer 1993, 67:1316-1323.

27. Hembruff SL, Laberge ML, Villeneuve DJ, Guo B, Veitch Z, Cecchetto M Parissenti AM: Role of drug transporters and drug accumulation in the temporal acquisition of drug resistance. BMC Cancer 2008, 8:318.

28. Klein TE, Chang JT, Cho MK, Easton KL, Fergerson R, Hewett M, Lin Z, Liu Y, Liu S, Oliver DE, Rubin DL, Shafa F, Stuart JM, Altman RB: Integrating genotype and phenotype information: an overview of the PharmGKB project. Pharmacogenetics Research Network and Knowledge Base. Pharmacogenomics J 2001, 1:167-170.

29. Nikitin A, Egorov S, Daraselia N, Mazo I: Pathway studio-the analysis and navigation of molecular networks. Bioinformatics 2003, 19:2155-2157.

30. Joshi-Tope G, Gillespie M, Vastrik I, D'Eustachio P, Schmidt E, de BB, Jassal B, Gopinath GR, Wu GR, Matthews L, Lewis S, Birney E, Stein L: Reactome: a knowledgebase of biological pathways. Nucleic Acids Res 2005, 33:D428-D432.

31. Dahlquist KD, Salomonis N, Vranizan K, Lawlor SC, Conklin BR: GenMAPP, a new tool for viewing and analyzing microarray data on biological pathways. Nat Genet 2002, 31:19-20.

32. Dennis G Jr, Sherman BT, Hosack DA, Yang J, Gao W, Lane HC, Lempicki RA: DAVID: Database for Annotation, Visualization, and Integrated Discovery. Genome Biol 2003, 4:3.
33. Okabe M, Unno M, Harigae H, Kaku M, Okitsu Y, Sasaki T, Mizoi T, Shiiba K, Takanaga H, Terasaki T, Matsuno S, Sasaki I, Ito S, Abe T: Characterization of the organic cation transporter SLC22A16: a doxorubicin importer. Biochem Biophys Res Commun 2005, 333:754-762.

34. Schriner SE, Linford NJ, Martin GM, Treuting P, Ogburn CE, Emond M, Coskun PE, Ladiges W, Wolf N, Van RH, Wallace DC, Rabinovitch PS: Extension of murine life span by overexpression of catalase targeted to mitochondria. Science 2005, 308:1909-1911.

35. Jin Y, Penning TM: Aldo-keto reductases and bioactivation/detoxication. Annu Rev Pharmacol Toxicol 2007, 47:263-292.

36. Ohara H, Miyabe Y, Deyashiki Y, Matsuura K, Hara A: Reduction of drug ketones by dihydrodiol dehydrogenases, carbonyl reductase and aldehyde reductase of human liver. Biochem Pharmacol 1995, 50:221-227.

37. Martin HJ, Maser E: Role of human aldo-keto-reductase AKR1B10 in the protection against toxic aldehydes. Chem Biol Interact 2009, 178:145-150.

38. Qadir M, O'Loughlin KL, Fricke SM, Williamson NA, Greco WR, Minderman H, Baer MR: Cyclosporin A is a broad-spectrum multidrug resistance modulator. Clin Cancer Res 2005, 11:2320-2326.

39. Olson RD, Mushlin PS, Brenner DE, Fleischer S, Cusack BJ, Chang BK, Boucek RJ Jr: Doxorubicin cardiotoxicity may be caused by its metabolite, doxorubicinol. Proc Natl Acad Sci USA 1988, 85:3585-3589.

40. Mordente A, Minotti G, Martorana GE, Silvestrini A, Giardina B, Meucci E: Anthracycline secondary alcohol metabolite formation in human or rabbit heart: biochemical aspects and pharmacologic implications. Biochem Pharmacol 2003, 66:989-998.

41. Kapadia $L$, Elder MG: Flufenamic acid in treatment of primary spasmodic dysmenorrhoea. A double-blind crossover study. Lancet 1978, 1:348-350.

42. Eisenhart $C$ : The assumptions underlying the analysis of variance. Biometrics 1947, 3:1-21.

43. Saeed Al, Sharov V, White J, Li J, Liang W, Bhagabati N, Braisted J, Klapa M, Currier T, Thiagarajan M, Sturn A, Snuffin M, Rezantsev A, Popov D, Ryltsov A, Kostukovich E, Borisovsky I, Liu Z, Vinsavich A, Trush V, Quackenbush J: TM4: a free, open-source system for microarray data management and analysis. Biotechniques 2003, 34:374-378.

44. Hembruff SL, Villeneuve DJ, Parissenti AM: The optimization of quantitative reverse transcription PCR for verification of CDNA microarray data. Anal Biochem 2005, 345:237-249.

45. Bustin SA, Benes V, Garson JA, Hellemans J, Huggett J, Kubista M, Mueller R, Nolan T, Pfaffl MW, Shipley GL, Vandesompele J, Wittwer CT: The MIQE guidelines: minimum information for publication of quantitative realtime PCR experiments. Clin Chem 2009, 55:611-622.

46. Lin HK, Steckelbroeck S, Fung KM, Jones AN, Penning TM: Characterization of a monoclonal antibody for human aldo-keto reductase AKR1C3 (type 2 3alpha-hydroxysteroid dehydrogenase/type 5 17beta-hydroxysteroid dehydrogenase); immunohistochemical detection in breast and prostate. Steroids 2004, 69:795-801.

47. Boger DL, Fink BE, Brunette SR, Tse WC, Hedrick MP: A simple, highresolution method for establishing DNA binding affinity and sequence selectivity. J Am Chem Soc 2001, 123:5878-5891.

48. Chadderton A, Villeneuve DJ, Gluck S, Kirwan-Rhude AF, Gannon BR, Blais $D E$, Parissenti AM: Role of specific apoptotic pathways in the restoration of paclitaxel-induced apoptosis by valspodar in doxorubicin-resistant MCF-7 breast cancer cells. Breast Cancer Res Treat 2000, 59:231-244.

49. Pollard JW, Walker JM: Animal Cell Culture. 5th edition. Clifton NJ: Humana Press; 1990.

doi:10.1186/1471-2407-12-381

Cite this article as: Heibein et al:: Role of aldo-keto reductases and other doxorubicin pharmacokinetic genes in doxorubicin resistance, DNA binding, and subcellular localization. BMC Cancer 2012 12:381. 